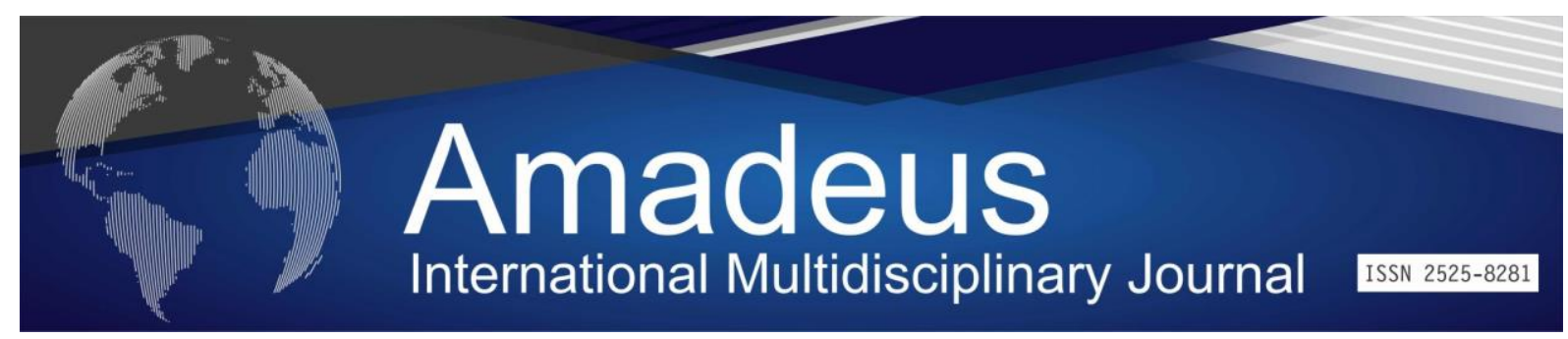

DOI: 10.14295/aimj.v5i9.134

\title{
Importance of Healthy Food for the Quality of Life of the Elderly
}

Carmelita Maria Silva Sousa ${ }^{1}$,

Thayna Bezerra de Luna ${ }^{2}$, Iasmin Belém Silva Queiroz ${ }^{3}$, Allex Alves Sobral de Sousa ${ }^{4}$,

Lina Maria Vidal Romão ${ }^{5}$,

Pedro Walisson Gomes

Feitosa $^{6}$,

Samyra Maria Lima

Sampaio $^{7}$,

José Leonardo Gomes

Coelho ${ }^{8}$,

Willma José de Santana,

Dayse Christina Rodrigues

Pereira Luz ${ }^{10}$
Abstract: Population aging is increasing at a rapid pace and it is a longing for today's society to grow healthy and with quality of life. The study provides a greater understanding of healthy eating that contributes to a better quality of life for the elderly. The study aims to analyze the importance of healthy eating for the elderly. It is understood that when combined with other activities the results are the best with regard to quality of life. The study is defined as an integrative review and qualitative approach. From the descriptors and the inclusion and exclusion criteria, thirty scientific articles were obtained and from these, ten were selected to compose the sample for the elaboration of this study and discuss the theme in focus. It is clear that healthy eating can and should favor the elderly with a substantial improvement in quality of life. It is necessary a nutritional monitoring with the elderly in order to contribute more and more to the quality of life and also subsidize the actions directed to this public.

Keywords: Healthy eating. Quality of life. Old man.

\footnotetext{
${ }^{1}$ Mestranda em Políticas Públicas pela Universidade Athenas College. ORCID http://orcid.org/0000-0002-2288-5554. email: carmelitasilva11@hotmail.com:

${ }^{2}$ Mestranda em Políticas Públicas pela Universidade Athenas College. ORCID http://orcid.org/0000-0003-1834-1623

${ }^{1}$ Mestranda em Políticas Públicas pela Universidade Athenas College. ORCID http://orcid.org/0000-0002-2288-5554 email: carmelitasilva11@hotmail.com

${ }_{2}$ Mestranda em Políticas Públicas pela Universidade Athenas College. ORCID http://orcid.org/0000-0003-1834-1623. email: thaynabezerra@hotmail.com;

${ }^{3}$ Mestranda em Políticas Públicas pela Universidade Athenas College. ORCID http://orcid.org/0000-0001-5237-9652. email: iasminbelém@hotmail.com;

${ }^{4}$ Mestrando em Políticas Públicas pela Universidade Athenas College. ORCID http://orcid.org/0000-0002-5237-6507. email: allexsobralfisio@ hotmail.com;

${ }_{5}^{5}$ Mestranda em Políticas Públicas pela Universidade Athenas College. ORCID http://orcid.org/0000-0002-3089-6608 email: linamariaromao@ hotmail.com;

${ }^{6}$ Acadêmico de Medicina pela Universidade Federal do Cariri. ORCID http://orcid.org/0000-0003-2320-8632. email: gomesfeitosa.walisson@outlook.com;

${ }^{7}$ Mestranda em Políticas Públicas pela Universidade Athenas College. ORCID http://orcid.org./0000-0003-0247-6814. email: samyrasampaio3@hotmail.com;

${ }^{8}$ Acadêmico do Curso de Farmácia do Centro Universitario de Juazeiro do Norte- UNIJUAZEIRO. ORCID:https://orcid.org/0000-00016028-0807. email: leonardo-coelho-10@hotmail.com;

${ }^{9}$ Pós Doutoranda em Ciências da Saúde - FMABC, Doutora em Ciências Biológicas pela UFPE e Docente do Centro Universitário de Juazeiro do Norte - UNIJUAZEIRO e Faculdade de Tecnologia - FATEC - CARIRI. ORCID: http://orcid.org/0000-0003-2733-2892 email:wjsantana@hotmail.com;

${ }^{10}$ Doutora em Ciências da Saúde pelo Centro Universitário de Saúde ABC, Pós - Doutoranda em Ciências da Saúde pelo Centro Universitário de Saúde ABC. Docente do Centro Universitario de Juazeiro do Norte- UNIJUAZEIRO. Grupo de Pesquisa do Conselho Nacional de Desenvolvimento Científico e Tecnológico - CNPq Diretório dos Grupos de Pesquisa no Brasil - DGP email: dayseluz.dcrp@gmail.com.
} 


\section{Importância da Alimentação Saudável para a Qualidade de Vida dos Idosos}

\begin{abstract}
Resumo: O envelhecimento populacional aumenta em ritmo acelerado e torna-se um anseio da sociedade atual envelhecer saudável e com qualidade de vida. $\mathrm{O}$ estudo proporciona um maior entendimento no que concerne uma alimentação saudável que contribua para uma melhor qualidade de vida dos idosos. $\mathrm{O}$ estudo tem por objetivo analisar a importância da alimentação saudável para os idosos. Entende- se que quando combinadas com outras atividades os resultados são dos melhores com relação à qualidade de vida. $\mathrm{O}$ estudo define-se como revisão integrativa e abordagem qualitativa. A partir dos descritores e dos critérios de inclusão e exclusão obteve-se trinta artigos científicos e, destes selecionou-se dez que compuseram a amostra para elaboração desse estudo e discorrem sobre o tema em foco. Percebe- se que uma alimentação saudável pode e deve favorecer aos idosos uma melhora substancial na qualidade de vida. Faz-se necessário um acompanhamento nutricional com os idosos no sentido de contribuir cada vez mais com a qualidade de vida e ainda subsidiar as ações direcionadas a esse público.
\end{abstract}

Palavras - chave: Alimentação saudável. Qualidade de vida. Idoso.

\section{Introdução}

O envelhecimento da população aumenta de maneira acentuada no nosso país e, mediante esse crescimento calcula-se que até o ano 2050, o Brasil será um país de idosos, superando assim o número de crianças e jovens, o que sinaliza para uma preocupação com a qualidade de vida dessa esfera da população (Sousa; Rodrigues, 2015). Dessa forma, envelhecer com qualidade de vida torna-se um anseio da sociedade atual (Pereira; Nogueira; Silva, 2015). É importante compreender que o prolongamento da vida é considerado uma conquista na medida em que soma qualidade aos anos a mais de existência (Confortin et al, 2017). Ressalta-se que a qualidade com que envelhecemos diz respeito às atitudes tomadas durante a vida, no entanto manter hábitos alimentares saudáveis no envelhecimento proporciona uma melhor qualidade de vida como também aspectos nutricionais (Toldra et al, 2014). Uma alimentação saudável envolve aspectos culturais e nutricionais, porém essas categorias convergem ou fazem uso do mesmo pensamento quando se refere do que é ou não bom para a saúde, tornando o tema complexo no tocante a análise de compreensão, pois nela se encontra diversos aspectos que envolvem o ser biopsicossocial (Tavares et al, 2017). 
Assim, a alimentação quando equilibrada e contendo todos os nutrientes necessários para o bom funcionamento do organismo idoso, pode contribuir atenuando os quadros inflamatórios e ainda reduzir o surgimento e desenvolvimento de patologias crônicas (Tavares et al, 2015).

Assim, faz-se necessário que o idoso se mantenha ativo e adote hábitos de vida saudáveis que proporcionem a manutenção da imunidade e também do vigor físico e mental, para garantir a saúde e bem estar (Filho, 2017).

A nutrição assume papel fundamental na modulação do declínio da idade, na origem das doenças associadas, assim como das funções estruturais e o aparecimento de deficiências (Assumpção et al, 2014). É importante motivar os idosos para uma atenção integral que não somente uma alimentação saudável, mas outras atividades que quando agregadas colaboram de forma positiva para uma vida com qualidade (Gomes; Soares; Gonçalves, 2016).

Sabe-se que a ingestão de uma alimentação saudável contribui de forma positiva na promoção da saúde, na prevenção de doenças, o que proporciona uma boa qualidade de vida e também um ritmo favorável no processo de envelhecimento (Deon et al, 2015; Martins, 2013).

Faz - se necessário uma avaliação nutricional com acompanhamento alimentar no sentido de proporcionar uma assistência adequada para os idosos e também sinalizar subsídios para o planejamento das ações voltadas para esse universo buscado sempre oferecer uma boa qualidade de vida.

\section{Metodologia}

Trata-se de uma Revisão da Literatura Integrativa, onde o levantamento dos artigos para construção deste estudo deu-se nas seguintes bases de dados: LILACS (LatinoAmericano e do Caribe em Ciência em Saúde), MEDLINE (Meidial Literatura Analyis and Retrieval System Online), SciELO ( Scientific Eletronic Litronic Library online) e PuBmed ( US National Librany of Medicine), com os seguintes descritores: Alimentação Saudável, Idoso, Qualidade de Vida.

$\mathrm{Na}$ seleção dos artigos, os critérios de inclusão foram: artigos disponíveis gratuitamente e na íntegra, nos idiomas português, inglês e espanhol de 2014 a 2019. Como critérios de exclusão optou-se por não utilizar teses, dissertações e monografias.

A busca pelos citados artigos se deu via on-line no período de outubro a novembro de 2019. A princípio foram encontrados trinta artigos. Após a leitura dos mesmos selecionou- 
se dez que compuseram a amostra para elaboração do estudo e que discorrem a cerca do tema em foco. Foi feita a análise descritiva dos estudos selecionados, buscando sempre estabelecer a síntese integrativa dos achados nos referidos estudos utilizados para tal fim.

\section{Resultados e Discussões}

Selecionados os artigos que atenderam aos critérios de inclusão estabelecidos, agrupou-se no quadro a seguir usando as categorias que discorrem sobre a importância da alimentação saudável para a qualidade de vida dos idosos.

Tabela 1 - Artigos que atenderam aos critérios de inclusão d estudo.

\begin{tabular}{|c|c|c|c|c|}
\hline $\begin{array}{l}\text { Autores/Ano da } \\
\text { Publicação }\end{array}$ & $\begin{array}{c}\text { Título da } \\
\text { Publicação }\end{array}$ & Revista & Objetivo & Resultado \\
\hline $\begin{array}{c}\text { Daniela de } \\
\text { Assumpção, } \\
\text { Semiramis M. A. } \\
\text { Domene, Regina } \\
\text { Mara Fisberg, } \\
\text { Marilisa B.de A. } \\
\text { Barros } \\
\text { ( 2014). }\end{array}$ & $\begin{array}{c}\text { Qualidade da dieta } \\
\text { e fatores } \\
\text { associados entre } \\
\text { idosos: estudo de } \\
\text { base populacional } \\
\text { em Campinas, São } \\
\text { Paulo, Brasil. }\end{array}$ & Cad. Saúde Pública. & $\begin{array}{c}\text { Avaliar a } \\
\text { qualidade da dieta } \\
\text { de idosos de } \\
\text { acordo com as } \\
\text { variáveis } \\
\text { socidemográficas, } \\
\text { comportamentos } \\
\text { relacionados à } \\
\text { saúde e } \\
\text { morbidades. }\end{array}$ & $\begin{array}{l}\text { Os achados sinalizam } \\
\text { para a necessidade de } \\
\text { melhora da qualidade } \\
\text { da dieta dos idosos } \\
\text { em geral e, de modo } \\
\text { particular para os } \\
\text { segmentos que } \\
\text { apresentam outros } \\
\text { comportamentos não } \\
\text { saudáveis. Apontam } \\
\text { também para a } \\
\text { relevância de } \\
\text { intervenções } \\
\text { integradas e não } \\
\text { focadas apenas em } \\
\text { um determinante. }\end{array}$ \\
\hline $\begin{array}{c}\text { Ana Paula } \\
\text { Gomes, Ana } \\
\text { Luiza Gonçalves } \\
\text { Soares, Helen } \\
\text { Gonçalves. } \\
\text { (2016) }\end{array}$ & $\begin{array}{c}\text { Baixa qualidade } \\
\text { da dieta de idosos: } \\
\text { estudo } \\
\text { populacional no } \\
\text { Sul do Brasil. }\end{array}$ & Ciênc. Saúde Coletiva & $\begin{array}{c}\text { Identificar os } \\
\text { fatores associados } \\
\text { à baixa qualidade } \\
\text { da dieta de idosos } \\
\text { da cidade de } \\
\text { Pelotas/RS. }\end{array}$ & $\begin{array}{l}\text { Os fatores associados } \\
\text { à dieta de baixa } \\
\text { qualidade foram sexo } \\
\text { masculino, idade } \\
\text { inferior a } 80 \text { anos, } \\
\text { baixa escolaridade, } \\
\text { falta de meios para } \\
\text { comprar os alimentos, } \\
\text { baixo peso, } \\
\text { problemas na boca ou } \\
\text { nos dentes e realizar } \\
\text { menos de quatro } \\
\text { refeições por dia. } \\
\text { Importantes barreiras } \\
\text { para o consumo de } \\
\text { uma dieta de boa } \\
\text { qualidade foram } \\
\text { identificadas nesse } \\
\text { estudo, ficando } \\
\text { evidente a } \\
\text { importância de se }\end{array}$ \\
\hline
\end{tabular}




\begin{tabular}{|c|c|c|c|c|}
\hline & & & & $\begin{array}{c}\text { considerar esses } \\
\text { aspectos na promoção } \\
\text { da alimentação } \\
\text { saudável em idosos. }\end{array}$ \\
\hline $\begin{array}{c}\text { Rúbia G. Deon, } \\
\text { Rosane D. da } \\
\text { Rosa, Vívian P. } \\
\text { S. Zaanardo, Vera } \\
\text { E. Closs, Carla } \\
\text { Helena A. } \\
\text { Schwanke. } \\
\text { (2015) }\end{array}$ & $\begin{array}{l}\text { Consumo de } \\
\text { alimentos dos } \\
\text { grupos que } \\
\text { compõem a } \\
\text { pirâmide } \\
\text { alimentar } \\
\text { americana por } \\
\text { idosos brasileiros: } \\
\text { uma revisão. }\end{array}$ & Ciência \& Saúde & $\begin{array}{l}\text { Apresentar uma } \\
\text { revisão literária } \\
\text { sobre as } \\
\text { evidências do } \\
\text { consumo de } \\
\text { alimentos dos } \\
\text { grupos que } \\
\text { compõem a } \\
\text { pirâmide } \\
\text { alimentar } \\
\text { americana para } \\
\text { idosos brasileiros. }\end{array}$ & $\begin{array}{l}\text { As pessoas do Brasil } \\
\text { de diferentes épocas e } \\
\text { regiões, o consumo } \\
\text { de alimentos } \\
\text { pertencentes a oito } \\
\text { dos nove grupos da } \\
\text { pirâmide alimentar } \\
\text { adaptada para a } \\
\text { população idosa } \\
\text { americana, cereais/ } \\
\text { pães/ tubérculos/ } \\
\text { raízes, hortaliças, } \\
\text { frutas, legumes, leite/ } \\
\text { derivados, carnes/ } \\
\text { ovos, açúcares/ doces } \\
\text { e água não é } \\
\text { adequada. As } \\
\text { evidências sinalizam } \\
\text { que o consumo, para } \\
\text { idosos brasileiros, dos } \\
\text { integrantes do grupo } \\
\text { da pirâmide alimentar } \\
\text { adaptada para a } \\
\text { população idosa } \\
\text { americana, de modo } \\
\text { geral, não se } \\
\text { apresenta adequada. }\end{array}$ \\
\hline $\begin{array}{c}\text { Jaqueline D. } \\
\text { Sousa, Marcos V. } \\
\text { Martins, } \\
\text { Fernanda S. } \\
\text { Franco, Karina O. } \\
\text { Martinha, } \\
\text { Adelson L. } \\
\text { Tinôco. } \\
\text { (2016). }\end{array}$ & $\begin{array}{l}\text { Padrão alimentar } \\
\text { de idosos: } \\
\text { caracterização e } \\
\text { associação com os } \\
\text { aspectos } \\
\text { socioeconômicos. }\end{array}$ & $\begin{array}{c}\text { Revista Bras. Geriatr } \\
\text { Gerontol. }\end{array}$ & $\begin{array}{c}\text { Verificar a } \\
\text { associação entre } \\
\text { padrões } \\
\text { alimentares e } \\
\text { aspectos } \\
\text { socioeconômicos } \\
\text { de idosos } \\
\text { atendidos pela } \\
\text { Estratégia Saúde } \\
\text { da Família no } \\
\text { município de } \\
\text { Viçosa, Minas } \\
\text { Gerais. }\end{array}$ & $\begin{array}{l}\text { O padrão alimentar } \\
\text { gordura e açúcar foi } \\
\text { maior no sexo } \\
\text { feminino e menor em } \\
\text { idosos que se } \\
\text { consideravam pardos. } \\
\text { No tocante ao padrão } \\
\text { balanceado, foi } \\
\text { inferior em sujeitos } \\
\text { pardos, casados, com } \\
\text { escolaridade de um a } \\
\text { quatro anos e da } \\
\text { classe econômica } \\
\text { CDE. Frutas e peixes } \\
\text { se apresenta menos } \\
\text { consumidos pelos } \\
\text { idosos cuja } \\
\text { escolaridade é de um } \\
\text { a quatro anos, ao } \\
\text { contrário do } \\
\text { observado naqueles } \\
\text { com cinco anos ou } \\
\text { mais de estudo. }\end{array}$ \\
\hline $\begin{array}{l}\text { Ariadne Cecílio, } \\
\text { Julicristie } \\
\text { Machado de } \\
\text { Oliveira. } \\
\text { (2015) }\end{array}$ & $\begin{array}{c}\text { Educação } \\
\text { Nutricional para } \\
\text { idosos } \\
\text { institucionalizados } \\
\text { no Recanto Nossa }\end{array}$ & $\begin{array}{l}\text { Estudo. Interdiscipl. } \\
\text { Envelhece. }\end{array}$ & $\begin{array}{c}\text { Promover, por } \\
\text { meio de atividades } \\
\text { de Educação } \\
\text { Nutricional, } \\
\text { hábitos }\end{array}$ & $\begin{array}{l}\text { A educação alimentar } \\
\text { proporciona } \\
\text { mudanças mesmo que } \\
\text { sutis, embora } \\
\text { importantes logo nas }\end{array}$ \\
\hline
\end{tabular}




\begin{tabular}{|c|c|c|c|c|}
\hline & $\begin{array}{l}\text { Senhora do } \\
\text { Rosário, em } \\
\text { Limeira, SP. }\end{array}$ & & $\begin{array}{l}\text { alimentares } \\
\text { saudáveis em um } \\
\text { grupo de idosos } \\
\text { institucionalizados } \\
\text { da cidade de } \\
\text { Limeira. }\end{array}$ & $\begin{array}{l}\text { primeiras semanas } \\
\text { logo após as } \\
\text { atividades realizadas. }\end{array}$ \\
\hline $\begin{array}{l}\text { Erielma P. de } \\
\text { Lima, Erika dos } \\
\text { Santos L. Maia, } \\
\text { Cladilene da Luz } \\
\text { F. Costa, Jessica } \\
\text { E. L. do Bu, } \\
\text { Arthur H.D } \\
\text { Barbosa. } \\
\text { (S/d). }\end{array}$ & $\begin{array}{l}\text { A importância da } \\
\text { orientação } \\
\text { nutricional para a } \\
\text { terceira Idade }\end{array}$ & $\begin{array}{l}\text { Editorarealize.com.br/ } \\
\text { revistas/ Conbracis/ } \\
\text { TRABALHOS. }\end{array}$ & $\begin{array}{c}\text { Mostrar os } \\
\text { principais estudos } \\
\text { sobre o } \\
\text { envelhecimento } \\
\text { humano e } \\
\text { qualidade de vida } \\
\text { nutricional. }\end{array}$ & $\begin{array}{c}\text { A ingestão de } \\
\text { alimentos saudáveis } \\
\text { contribui } \\
\text { significativamente } \\
\text { para a saúde do } \\
\text { indivíduo na terceira } \\
\text { idade, e reduz } \\
\text { possíveis alterações } \\
\text { patológicas no } \\
\text { processo de } \\
\text { envelhecimento. }\end{array}$ \\
\hline $\begin{array}{l}\text { Thaynã R. Flores, } \\
\text { Ana P. Gomes, } \\
\text { Ana L. G. Soares, } \\
\text { Bruno P. Nunes, } \\
\text { Maria C. F. } \\
\text { Assunção, Helen } \\
\text { Gonçalves andrea } \\
\text { D. Bertoldi. } \\
\text { (2014) }\end{array}$ & $\begin{array}{l}\text { Aconselhamento } \\
\text { por profissionais } \\
\text { de saúde e } \\
\text { comportamentos } \\
\text { saudáveis entre } \\
\text { idosos: estudo de } \\
\text { base em Pelotas, } \\
\text { Sul do Brasil. }\end{array}$ & $\begin{array}{l}\text { Epidemiol. Serv. } \\
\text { Saúde. }\end{array}$ & $\begin{array}{c}\text { Analisar a } \\
\text { associação entre } \\
\text { recebimento de } \\
\text { orientações por } \\
\text { profissionais de } \\
\text { saúde e } \\
\text { comportamentos } \\
\text { saudáveis entre } \\
\text { idosos. }\end{array}$ & $\begin{array}{c}\text { Os idosos que } \\
\text { receberam } \\
\text { orientações dos } \\
\text { profissionais de saúde } \\
\text { relataram redução no } \\
\text { consumo do sal, } \\
\text { açúcar e doces e, } \\
\text { também maior a } \\
\text { prática de atividade } \\
\text { física, em } \\
\text { comparação aos } \\
\text { idosos desprovidos } \\
\text { das orientações. }\end{array}$ \\
\hline $\begin{array}{l}\text { Silvia Martinez } \\
\text { (2013) }\end{array}$ & $\begin{array}{c}\text { A nutrição e } \\
\text { alimentação como } \\
\text { pilares dos } \\
\text { programas de } \\
\text { promoção de } \\
\text { saúde e qualidade } \\
\text { de vida nas } \\
\text { organizações. }\end{array}$ & $\mathrm{O} \mathrm{Mu}$ & $\begin{array}{c}\text { Relacionar as } \\
\text { questões } \\
\text { nutricionais com } \\
\text { os parâmetros } \\
\text { para elaboração de } \\
\text { programas de } \\
\text { qualidade de vida. }\end{array}$ & $\begin{array}{l}\text { Percebe-se que a } \\
\text { alimentação pode e } \\
\text { deve contribuir na } \\
\text { melhora da qualidade } \\
\text { de vida tanto física } \\
\text { quanto mental e } \\
\text { social. }\end{array}$ \\
\hline $\begin{array}{c}\text { Janyne V. Melo, } \\
\text { Ana L. M. } \\
\text { Santos, } \\
\text { Alexandre A. A. } \\
\text { de Rezende, } \\
\text { Luciana K. } \\
\text { Calábria. } \\
\text { ( 2017). }\end{array}$ & $\begin{array}{c}\text { Hábitos } \\
\text { alimentares dos } \\
\text { idosos atendidos } \\
\text { nos programas de } \\
\text { saúde da família } \\
\text { (PSF) em } \\
\text { Ituiutaba- MG. }\end{array}$ & Revista MedSaúde. & $\begin{array}{l}\text { Analisar a atual } \\
\text { situação da } \\
\text { nutrição dos } \\
\text { idosos residentes } \\
\text { no município de } \\
\text { Ituiutaba- MG, } \\
\text { considerando os } \\
\text { "10 passos para } \\
\text { alimentação } \\
\text { saudável para as } \\
\text { pessoas idosas. }\end{array}$ & $\begin{array}{l}\text { O estudo constata que } \\
\text { os hábitos alimentares } \\
\text { saudáveis são } \\
\text { importantes e devem } \\
\text { ser seguidos uma vez } \\
\text { que contribui } \\
\text { positivamente para a } \\
\text { qualidade de vida dos } \\
\text { idosos. }\end{array}$ \\
\hline $\begin{array}{c}\text { Sara Franco D. } \\
\text { Heitor, Leiner R. } \\
\text { Rodrigues, } \\
\text { Darlene M. dos } \\
\text { Santos Tavares. } \\
\text { (2013) }\end{array}$ & $\begin{array}{c}\text { Prevalência da } \\
\text { adequação e } \\
\text { alimentação } \\
\text { saudável de idosos } \\
\text { residentes na zona } \\
\text { rural. }\end{array}$ & $\begin{array}{l}\text { Texto \& Contexto- } \\
\text { enfermagem. }\end{array}$ & $\begin{array}{l}\text { Determinar a } \\
\text { prevalência da } \\
\text { adequação do } \\
\text { Guia "10 passos } \\
\text { para uma } \\
\text { alimentação } \\
\text { saudável para a } \\
\text { pessoa idosa" } \\
\text { além de } \\
\text { caracterizar o }\end{array}$ & $\begin{array}{c}\text { A análise dos dados } \\
\text { constitui distribuição } \\
\text { de frequência } \\
\text { simples. Nenhum } \\
\text { idoso segui todos os } \\
\text { passos. O consumo de } \\
\text { sal se apresenta como } \\
\text { maior segmento e, o } \\
\text { de carne e leite como } \\
\text { menor. }\end{array}$ \\
\hline
\end{tabular}


Fonte: Dados da Pesquisa, 2020.

Em relação à importância da alimentação saudável para a qualidade de vida dos idosos, apresenta-se a seguir a discussão entre os autores estudados.

Uma alimentação balanceada para os idosos faz-se necessário para melhorar a qualidade da dieta de modo geral e, em especial para os segmentos que apresentam comportamentos nada saudáveis. O estudo sinaliza também para uma intervenção integrada e não focada apenas em uma variável (Sousa et al, 2016; Cicílio; Oliveira, 2015).

Percebe-se que as pessoas idosas do Brasil de diferentes épocas e regiões, o consumo de alimentos pertencentes a oito dos nove grupos da pirâmide adaptada para os idosos americanos (cereais, pães, tubérculos, raízes, hortaliças, leite e derivados, carnes, ovos, açúcares, doces) e consumo de água não é adequado. Ressalta-se que o consumo de açúcar e gordura foi maior nas idosas, enquanto nos idosos foi menos expressivo (Borges; Seide, 2014.

O padrão alimentação balanceada apresenta-se menor em sujeitos casados, com um a quatro anos de escolaridade. Entretanto o consumo de frutas e peixes pelos idosos é menor e têm também de um a quatro anos de escolaridade quando comparados com os que apresentam cinco anos ou mais de escolaridade (Cicílio; Oliveira, 2015; Borges; Seide, 2014; Lima et al, 2020).

A educação alimentar propicia mudanças relevantes mesmo que pouco visíveis, porém torna-se importante para os idosos que participaram das atividades trabalhadas nesse sentido (Flores et al, 2018)

Colocando em pauta os idosos do sexo masculino, percebe-se o fortalecimento do repertório comportamental destes, da prática de exercícios físicos, como também de um padrão alimentar adequado (Martinez, 2013).

O hábito de ingestão de alimentos saudáveis contribui de modo significativo para a saúde do indivíduo na terceira idade e diminui possíveis mudanças relacionadas a doenças no decorrer do envelhecimento (Franco; Rodrigues; Tavares, 2013).

De acordo com os profissionais de saúde que orientam os idosos no que diz respeito aos hábitos para uma boa alimentação, constata-se que os idosos que receberam as 
orientações, reduziram o consumo do sal, carnes e doces e acentuaram a prática de exercícios físicos, quando comparados aos idosos que não receberam as orientações (Franco;Rodrigues; Tavares, 2013).

Sabe-se que a alimentação saudável pode e deve contribuir para a melhor qualidade de vida tanto física quanto mental e social (Flores et al, 2018). É notório que os hábitos alimentares saudáveis, contribuem significativamente para a qualidade de vida dos idosos (Martinez, 2013). 亡̀ importante ressaltar que nenhum idoso segue todos os passos preconizados no guia alimentar. $\mathrm{O}$ consumo de sal continua maior enquanto a carne e o leite menor (Franco; Rodrigues; Tavares, 2013).

\section{Considerações Finais}

Percebeu-se através do estudo que uma alimentação saudável atua como um efeito promotor de qualidade de vida contra o desenvolvimento e o surgimento de danos relacionados com a nutrição. Com relação aos idosos uma alimentação adequada deve proporcionar mais capacidades funcionais e diminuir as doenças. Contudo faz-se necessário o acompanhamento por parte dos profissionais de saúde, através de possíveis riscos já existentes no sentido de orientar os idosos para uma alimentação saudável, devendo ser constante seja na instituição seja na família, na busca de proporcionar uma qualidade de vida satisfatória tão almejada por todos.

\section{Referências}

Assumpção, D. et al (2014). Qualidade da dieta e fatores associados entre idosos: estudo de base populacional em Campinas, São Paulo, Brasil. Cad. Saúde Pública, Rio de Janeiro, 30 (8): 1680- 1694. http://dx.doi.org/10.1590/0102-311X0000113

Borges, L. M., Seidi, E. M. F. (2014) Hábitos saudáveis na velhice: efeitos de uma intervenção psicoeducativa com homens idosos. Psic. Saúde\& Doenças. Vol.15 n. 2 Lisboa. ISSN: 1645-0086.

Cicílio, A., Oliveira, J. M. (2015). Educação nutricional para idosos institucionalizados no Recanto Nossa Senhora do Rosário em Limeira, SP. Estud. Interdiscipl. Envelhec, Porto Alegre, v 20 n.2 p 413 - 426.

Confortin, S. C. et al (2017). Condições de vida de idosos: resultados do estudo de coorte Epifloripa Idoso. Epidemiol. Serv. Saúde. V.26 n 2. Brasília. 
Deon, R. G. et al (2015). Consumo de alimentos dos grupos que compõem a pirâmide alimentar americana por idosos brasileiros: uma revisão. Ciência \& Saúde. V.8 n.1. Doi: http://dx.doi.org/10.15448/1983-652X

Filho, P. R. P. (2017). Importância da musculação na terceira idade. Revista Científica Multidisciplinar Núcleo de Conhecimento. Edição 03. Ano 02, vol.1, pp 391-406. ISSN: 2448-0959.

Flores, T. R. et al (2018). Aconselhamento por profissionais de saúde e comportamentos saudáveis entre idosos: estudo de base população em Pelotas, Sul do Brasil. Epidemiol. Serv. Saúde vol. 27 no. 1 Brasília. http:// dx. Doi. Org / 10. 523/ s1679 - 49742018000100012

Franco, S. D. H, Rodrigues, L. R., Tavares, D. M. S. (2013). Prevalência da adequação e alimentação saudável de idosos residentes na zona rural. Texto e Contexto- enferma. Vol.22 no 1 Florianópolis.

Gomes, A. P., Soares, A. L. G., Gonçalves, H. (2016). Baixa qualidade da dieta de idosos: estudo de base populacional no Sul do Brasil. Ciênc. Saúde Colet. 21 (11). https:// doi.org/10.1590/1413812320152111.17502015

Lima, E. P. et al (2020). A importância da orientação nutricional para a terceira idade. II CONBRACIS .editorarealize.com.br/revistas/conbracis/trabalhos/trabalho_VO71_MD4_SA6_IDS12_27042 017181215,pdf s/d

Martinez, S. (2013). A nutrição e alimentação como pilares dos programas de promoção de saúde e qualidade de vida nas organizações. O Mundo da Saúde. São Paulo, 37 (2): 201 207.

Pereira, S. D., Nogueira, D. A. J., Silva, B. A. C. (2015). Qualidade de vida e situação de idosos: um estudo de base populacional no Sertão Central do Ceará. Revista Brasileira de Geriatria e Gerontologia, Rio de Janeiro, 18 (4): 893 - 908. DOI: HTTP://dx.doi.org/10. 1590/1809-9823.201514123.

Sousa, A. Q. J., Rodrigues, N. R. (2015) Gestão da qualidade de vida dos idosos frequentadores do centro de convivência e apoio ao idoso Casa de Clara. Revista da FATEC Zona Sul, V.1, n³. www. Revistarefas.com.br/index.php/REVIFATEXCIS/ article/view/23.pdf

Sousa, J. D. et al (2016). Padrão alimentar de idosos: caracterização e associação com aspectos econômicos. Revista Bras.Geriatr. Gerontol, rio de Janeiro, 19 (2): 970-977. http://dx. Doi. Org/10.1590/1981-22562016019-160035.

Tavares, E. L. et al (2015). Avaliação nutricional de idosos: desafios da atualidade. Revista Brasileira Geriatri. Gerontol. Rio de Janeiro, 18 (3) 643-650. http://dx.doi,org/10.1590/18099823.2015.142.49

Tavares, R. E. et al (2017). Envelhecimento Saudável na perspectiva de idosos: uma revisão integrativa. Revista Bras Geriatr. Gerontol, Rio de Janeiro, 20(6):889-900. http: //dx.doi.org/10.1590/1981-22562017020-170091 
Toldrá, C. R. et al (2014). Promoção da saúde e da qualidade de vida com idosos por meio de práticas corporais. $O$ Mundo da Saúde. São Paulo, 38(2):159-169. DOI: 105343/01/047809.20143802159168.

\section{How to cite this article (APA format):}

Sousa, Carmelita Maria Silva; Luna, Thayna Bezerra de; Queiroz, Iasmin Belém Silva; Sousa, Allex Alves Sobral de; Romão, Lina Maria Vidal; Feitosa, Pedro Walisson Gomes; Sampaio, Samyra Maria Lima; Coelho, José Leonardo Gomes; Santana, Willma José de; Luz, Dayse Christina Rodrigues Pereira (2020). Importance of Healthy Food for the Quality of Life of the Elderly. Am. In. Mult. J., Jul to Out. (9) 5, 74-83.

Received: 08/09/2020

Accepted: 08/11/2020 